\title{
Buscando datos e indicadores para evaluar los servicios de atención temprana
}

\author{
Searching for data and indicators for \\ evaluating early childhood intervention \\ services
}

\section{Resumen}

La búsqueda de indicadores, de datos o referencias que permitan medir lo más objetivamente posible si los servicios de atención temprana cumplen adecuadamente su misión asistencial ha ido adquiriendo cada vez mayor relevancia en dicho ámbito, de forma correlativa con la extraordinaria expansión de las redes de centros y servicios en las diferentes comunidades autónomas de España. Se expone el sistema de indicadores que se ha utilizado para el seguimiento de tres unidades experimentales de la Red Gallega de Atención Temprana, señalando en este artículo la perspectiva y valoración realizada por sus profesionales.

\section{Palabras clave}

Atención temprana, evaluación, datos, indicadores.

\begin{abstract}
The search for indicators, data or references to measure, as objectively as possible, if services adequately fulfill its assistance mission, has gained increasing importance in the field of early intervention, correlatively with the extraordinary expansion of networks and service centers in the different autonomous communities. The system of indicators used to monitor three experimental units of the Galician Network of Early Intervention is exposed, noting in this article the perspective and the assessment performed by their professionals.
\end{abstract}

\section{Keywords}

Early childhood intervention, evaluation, data, indicators.
Jaime Ponte Mittelbrunn

$<$ jaime.ponte.mittelbrunn@xunta. es>

Xunta de Galicia

\author{
Para citar: \\ Ponte, J. (20I7): "Buscando datos e \\ indicadores para evaluar los servicios \\ de atención temprana”. Revista \\ Española de Discapacidad, 5 (I): \\ 77-98. \\ Doi: <https://doi.org/IO. 5569/2340- \\ 5104.05.01.05>
}

Fecha de recepción: 06-09-2016 Fecha de aceptación: IO-03-20I7 


\section{Introducción}

La sociedad de hoy reclama cada vez una mayor transparencia en la justificación y gestión del gasto público y, en ese sentido, hace tiempo que se ha impuesto la necesidad de contar con datos e indicadores que permitan valorar la calidad de los servicios y documentar la toma de decisiones respecto a su utilidad. Esta evaluación se hace especialmente compleja en algunos campos de los servicios sociales (Red de consultoría social, 20I0)

La búsqueda de indicadores, de datos o referencias que permitan medir lo más objetivamente posible si los servicios de atención temprana cumplen adecuadamente su misión asistencial ha ido adquiriendo cada vez mayor relieve, de forma correlativa a la expansión de la prestación. En España la extensión de la red de centros recibió un enorme impulso con la elaboración del Libro Blanco de la Atención Temprana (GAT, 2000), un documento de consenso profesional que fue respaldado por importantes organizaciones del tercer sector y por el conjunto de las administraciones públicas con la mediación del Real Patronato sobre Discapacidad.

Siguiendo los principios del Libro Blanco pueden encontrarse antecedentes de esta preocupación en documentos de 'orientación' sobre la calidad de los servicios cómo los elaborados por FEAPS (200I), en la Guía de Estándares de Calidad y Formulario de Autoevaluación (Ponte et al., 2004) o en las Recomendaciones Técnicas para el desarrollo de la Atención Temprana (GAT, 2005 ). Estos documentos pretendían servir como un primer marco de referencia para guiar el previsible desarrollo de una red estatal de Centros de Desarrollo Infantil y Atención Temprana (CDIAT) y tenían por lo tanto un fuerte carácter propositivo, de recogida y sistematización de buenas prácticas, centrándose sobre todo en señalar las diferentes dimensiones de actuación, unos contenidos comunes, unas actividades básicas y un modo de proceder por los servicios.
En esa época, pero ya con una finalidad más retrospectiva y de control de la práctica concreta, se elaboró también para el Instituto Catalán de Servicios Sociales (ICASS) por parte de la Fundación Avedis Donabedian y de la Unión de Centros de Atención Precoz (UCCAP) un sistema inicial de estándares para la evaluación externa de la calidad de los centros de desarrollo infantil y atención precoz (CDIAP) de la red catalana, con amplio soporte de datos (Giné, 2002). Este sistema ha ido evolucionando después hasta la reciente publicación del proyecto de Orden de acreditación de los Servicios de Atención Precoz y procedimiento de autorización de las entidades evaluadoras (Generalitat de Catalunya, 20I 5). En otras comunidades autónomas la expansión de la atención temprana ha dado lugar asimismo a una amplia normativa de autorización y acreditación de centros basada principalmente en condiciones de carácter estructural: infraestructura de los centros, materiales, plantillas de los servicios, principios generales de actuación o requisitos de acceso (Agencia de Calidad Sanitaria de Andalucía, 20I2). Desde otra perspectiva, vinculada a la calidad percibida, cabe destacar también las investigaciones realizadas por García Sánchez (García Sánchez et al., 2008) y Romero (20I I) en centros de atención temprana de Murcia y Málaga. El trabajo de García Sánchez está orientado a profundizar en la importancia que dan los padres a las distintas dimensiones del proceso de atención y el de Romero ha facilitado el diseño de un cuestionario de 39 ítems para conocer la satisfacción de las familias denominado Inventario de Calidad en los Centros de Atención Infantil Temprana (ICCAIT).

En esta línea de preocupación por avanzar y profundizar en la planificación, organización y evaluación de los servicios, aunque desde una realidad asistencial todavía en ciernes como la nueva Red Gallega de Atención Temprana (Decreto I 83/20I3, de 5 de diciembre, DOG 23/I2/20I3) (Xunta de Galicia, 20I3), se inscribe también el compromiso asumido por la Asociación Galega de Atención Temperá (AGAT) con la Xunta de Galicia para impulsar 
una experiencia asistencial y de investigación que, además de cubrir huecos asistenciales, ofreciera datos para el desarrollo de dicha Red. Así, a partir de 2009 y mediante convenio con la Consejería de Política Social, AGAT implantó en tres comarcas de Galicia (Deza, Salnés y Bergantiños) tres unidades piloto, las Unidades de Desarrollo Infantil y Apoyo Familiar (en adelante UDIAF), con un doble objetivo:

I. Cubrir el vacío asistencial existente en las respectivas comarcas mediante dispositivos comunitarios públicos y gratuitos, de carácter preventivo asistencial y dirigidos a proporcionar apoyos a los niños/as, a las familias y a los recursos del contorno.

2. Desarrollar tareas de evaluación y recogida de datos que permitieran realizar un estudio cuantitativo y cualitativo de la demanda efectiva en un ámbito territorial definido, experimentar procesos y metodologías, obtener resultados e investigar los niveles de satisfacción de las familias, contribuyendo a perfilar un dispositivo tipo con los servicios más necesarios, los indicadores de seguimiento de su gestión y los perfiles formativos de los profesionales.

Una parte sustancial de esta segunda tarea se ha cumplido mediante la remisión a la Xunta de Galicia a principios de 2016 del informe técnico UDIAFS. Servizos comunitarios de Atención Temperá (Ponte et al., 2016) en el que se realiza una reflexión global sobre el período 2009-20I6 y se llega a diversas conclusiones y recomendaciones para el desarrollo de la Red Gallega.

En este artículo ofreceremos una parte de dicho informe técnico: se describen los indicadores de seguimiento de la gestión que se han utilizado para registrar y valorar el trabajo de las UDIAF y se destacan aquellos que al cabo de los años han seleccionado los propios profesionales por considerarlos de mayor interés para hacer un seguimiento de su trabajo. Esperamos que tanto el cuadro general de indicadores utilizados para evaluar los servicios como su cualificación por parte de los mismos profesionales que los han utilizado puedan ser de utilidad para otras experiencias.

\section{Material y método}

Dentro de su diseño como unidades piloto las UDIAF se configuran como Servicios Sociales Comunitarios Específicos de Atención Temprana de acuerdo con la legislación vigente en Galicia ${ }^{\mathrm{I}}$ $y$, en cuanto a los principios de actuación y el enfoque de trabajo, siguen las orientaciones de los documentos de consenso profesional de nuestro entorno (Guralnick, I997; GAT, 2000; Dunst, 2004; Soriano, 2005; Soriano y Kyriazopoulou, 20I0; Moore, 2008). Las comarcas elegidas para llevar a cabo el proyecto fueron la comarca del Deza y la comarca del Salnés en la provincia de Pontevedra, a partir de junio de 2009, y la de Bergantiños en la provincia de A Coruña, a partir de 20 I $4 .^{2}$ Tres comarcas en las cuales no había servicios de atención temprana y con características demográficas diferentes. La comarca del Deza es una extensa comarca rural del centro de Galicia, con una población en torno a los 45.000 habitantes y cabecera en Lalín $( \pm 20.000$ h.). Mientras que el Salnés es un territorio costero con más de I००.০00 h., una de las zonas de Galicia más densamente poblada, con cabecera en Vilagarcía de Arousa ( \pm 37.000 h.). Y Bergantiños, por su parte, es una comarca que ocupa una zona intermedia entre el área metropolitana de A Coruña y el área marítimo rural de la Costa da Morte. Su capital es Carballo que cuenta con $\pm 3 \mathrm{I} .000 \mathrm{~h}$.

I. Decreto I $83 / 2013$ por el que se crea la Red Gallega de Atención Temprana. 


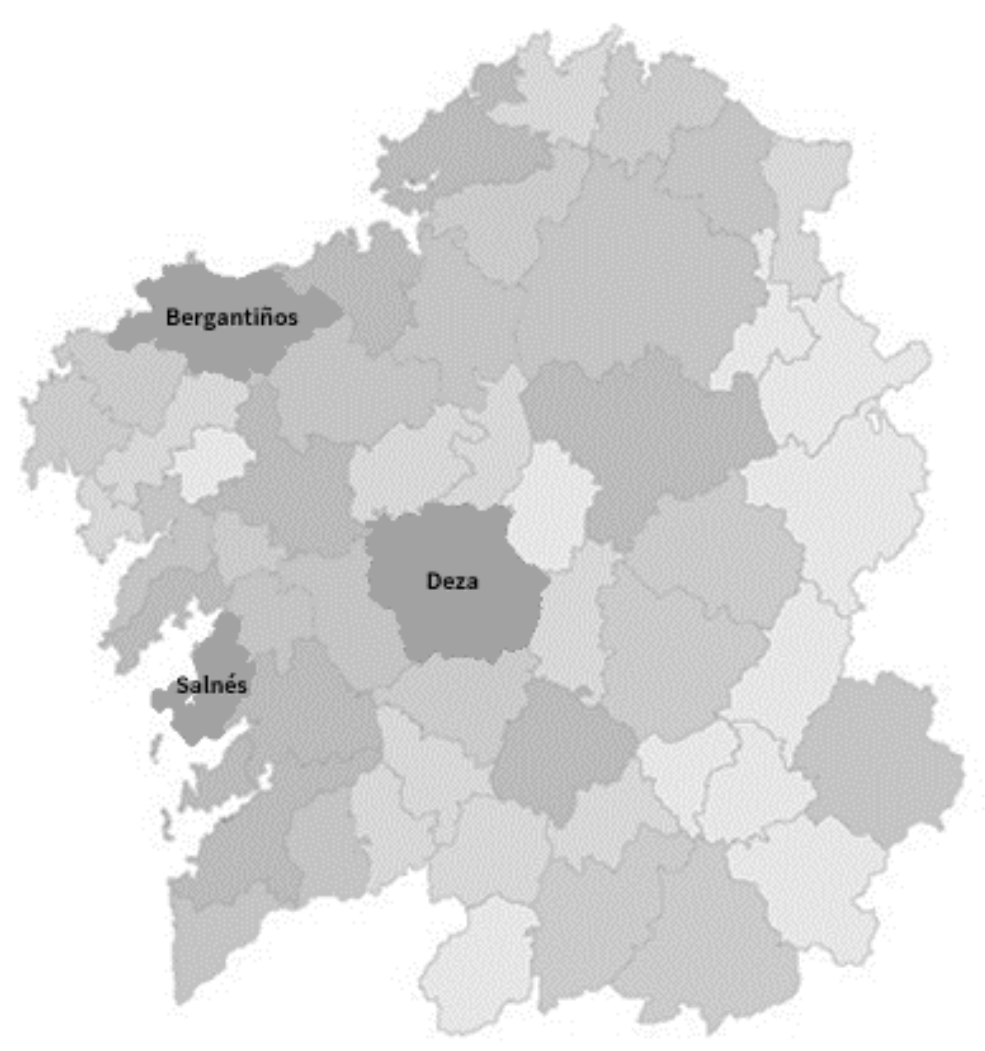

Fuente: elaboración propia.

Las UDIAF están localizadas en las cabeceras de sus respectivas comarcas. Se trata de centros situados sobre espacios cedidos por las administraciones locales: ayuntamientos de Lalín, Vilagarcía de Arousa y Carballo. Las UDIAF abren unas 44 horas semanales, de lunes a viernes en horario de mañana y tarde. Cada UDIAF alberga un pequeño equipo interdisciplinario con un psicólogo o pedagogo, coordinador del servicio y especializado en desarrollo infantil, atención temprana y apoyo familiar, dos terapeutas especializados en desarrollo infantil y atención temprana (terapeuta/ psicocomotricista/ logopeda) y un auxiliar administrativo. La mayoría de los profesionales están hoy contratados a tiempo completo, excepto los administrativos que trabajan ${ }_{5}$ horas por semana.
El proceso de actuación de las UDIAF sigue, en el trabajo de caso, las fases recomendadas por el Libro Blanco: consulta de acogida, valoración interdisciplinaria, entrevista de devolución y plan personalizado de intervención. Las UDIAF colaboran también en actividades comunitarias de prevención primaria y secundaria que se realizan en su territorio: sensibilización, prácticas de crianza, cursos de formación, etc.

Todos los equipos tuvieron un proceso de tutoría inicial de seis meses mediante participación de expertos de la AGAT en el arranque y desarrollo de las tareas iniciales. Posteriormente, las unidades contaron con el apoyo y orientación de un coordinador externo de la AGAT encargado de la supervisión del convenio, el ajuste a sus objetivos, la formación continua de 
los profesionales y la dirección del proceso de evaluación.

La financiación corre a cargo de la Xunta de Galicia, Consejería de Política Social, mediante convenio anual renovable con la Asociación Galega de Atención Temperá (AGAT). La cuantía inicial en 2009 , en el que se implantan las dos primeras unidades, fue de $200.000 € \mathrm{y}$ la prevista para el ejercicio 2016 , con tres, es de $300.000 €$.

Para registrar y poder dar cuenta del trabajo realizado, así como para establecer líneas de evaluación, se diseñó un modelo de memorias e indicadores ${ }^{2}$ que permitiera realizar el seguimiento de la actividad, objetivos y funciones del servicio y conocer la opinión de las familias. También se ha procurado aprovechar y buscar coincidencias con las propuestas de otras comunidades autónomas cuya legislación se revisó a través de la base de datos legislativa del Centro Español de Documentación sobre Discapacidad. Los 37 indicadores del cuadro inicial responden a la combinación de tres perspectivas habituales de evaluación (Donabedian, I988; VV.AA., I999): la estructural, la formativa y la sumativa.
El aspecto sumativo se ha subdividido para diferenciar tres dimensiones diferentes de resultados: los que nos permiten conocer mejor la población servida por las UDIAF (indicadores de alcance), los que examinan la actividad de cada unidad en términos cuantitativos $\left(\mathrm{n}^{\circ}\right.$ de sesiones realizadas) y los que nos ofrecen datos sobre el ajuste entre la demanda y los medios de que se disponen. A continuación, se exponen las cinco dimensiones de análisis con sus correspondientes resultados desglosados por cada UDIAF.

a. Indicadores de alcance: diseñados para estudiar la cobertura de la población diana de las UDIAF (indicador CI) y algunos de los datos que son más relevantes para la planificación asistencial: la tipología de la demanda en el momento de entrar en las unidades $\left(\mathrm{C}_{2}\right)$; los perfiles diagnósticos que, al finalizar la intervención, registran los profesionales siguiendo la clasificación diagnóstica ODAT $\left(\mathrm{C}_{3}\right)$ y el porcentaje de población que tiene reconocida discapacidad y dependencia $\left(\mathrm{C}_{4}\right)$. También se incluye la distribución por sexo $\left(\mathrm{C}_{5}\right)$ y la edad de los niños en el momento en que las familias hacen la solicitud de atención (C6).

\footnotetext{
2. Para una referencia más amplia consultar el informe técnico UDIAF. Servizos comunitarios de Atención Temperá, remitido a la Xunta de Galicia en 2016 por la Asociación Galega de Atención Temperá: http://atenciontemprana.com/wp-content/ uploads/20I 6/06/UDIAFS-SERVIZOS-COMUNITARIOS-AT. pdf.
} 
Tabla 1. Indicadores de alcance

Indicador C.1 Población 0-6 atendida

Relaciona el $\mathrm{n}^{\circ}$ de niños/as atendidos cada año con el $\mathrm{n}^{\circ}$ de niños/as de 0 a 6 años en cada comarca

\begin{tabular}{|l|c|c|c|c|c|c|}
\hline UDIAF & $\mathbf{2 0 1 0}$ & $\mathbf{2 0 1 1}$ & $\mathbf{2 0 1 2}$ & $\mathbf{2 0 1 3}$ & $\mathbf{2 0 1 4}$ & $\mathbf{2 0 1 5}$ \\
\hline Deza & 2,05 & 3,2 & 3,9 & 3,7 & 5,3 & 5,9 \\
\hline Salnés & 1,83 & 2,21 & 2,01 & 2 & 1,9 & 1,7 \\
\hline Bergantiños & & & & & & 2,4 \\
\hline
\end{tabular}

Indicador C.2 Tipología de la demanda

Relaciona el $n^{\circ}$ de niños atendidos con la tipología de problemas principales que presentan según la exposición de la familia en la consulta de acogida

\begin{tabular}{|l|c|c|c|}
\hline Tipología demanda & Deza & Salnés & Bergantiños \\
\hline Retraso evolutivo & 5,1 & 7,5 & 11,6 \\
\hline Probl. sindrómicos & 5,4 & 14,6 & 9,3 \\
\hline Lenguaje & 28,4 & 29,6 & 38,4 \\
\hline Cognitivo & 5,1 & 3,5 & 2,3 \\
\hline Hábitos & 8,6 & 5,9 & 9,3 \\
\hline Afectivo-emocional & 25,3 & 8,6 & 12,8 \\
\hline Conducta y atención & 16,3 & 24,3 & 12,8 \\
\hline Sensoriales & 0,4 & 1,1 & 2,3 \\
\hline Motrices & 4,7 & 4,6 & 1,2 \\
\hline Casos: 257 Deza, 371 Salnés, 86 Bergantiños \\
\hline
\end{tabular}

\begin{tabular}{|c|c|c|c|}
\hline \multicolumn{4}{|c|}{ Indicador C.3 Perfiles diagnósticos } \\
\hline \multicolumn{4}{|c|}{$\begin{array}{l}\text { Relaciona el } n^{\circ} \text { de niños/as atendidos con la tipología de problemas que presentan según la Organización } \\
\text { Diagnóstica en Atención Temprana (ODAT) Ejes IV, V, VI }\end{array}$} \\
\hline Perfil problemas & Deza & Salnés & Bergantiños \\
\hline Desarrollo motor & 3,9 & 5,4 & 5,8 \\
\hline Desarrollo visual & 4,3 & 9,2 & 0 \\
\hline Desarrollo auditivo & 1,6 & 4 & 1,2 \\
\hline Desarrollo psicomotor & 7,0 & 6,7 & 5,8 \\
\hline Retraso evolutivo & 5,1 & 17,3 & 14 \\
\hline Retraso mental & 3,5 & 1,3 & 1,2 \\
\hline Comunicación y lenguaje & 34,1 & 36,9 & 37,2 \\
\hline Psicosomáticos & 17,1 & 8,1 & 2,7 \\
\hline Afectivos & 19,1 & 15,4 & 11,6 \\
\hline Regulación y comportamiento & 11,1 & 26,4 & 24,4 \\
\hline Espectro autista & 3,5 & 7 & 7 \\
\hline Interacción en las familias & 16,7 & 35,8 & 12,8 \\
\hline Entorno con factores exclusión & 5,1 & 17 & 9,3 \\
\hline
\end{tabular}

Indicador C.4 Discapacidad/dependencia

Porcentaje de Población en situación de discapacidad/dependencia

$11 \%$ según el estudio realizado con los datos 2009-2011

\section{Indicador C.5 Sexo}

Porcentaje de niños/as atendidos según sexo

\begin{tabular}{|lc|c|c|c|c|c|c|}
\hline UDIAF & & $\mathbf{2 0 1 0}$ & $\mathbf{2 0 1 1}$ & $\mathbf{2 0 1 2}$ & $\mathbf{2 0 1 3}$ & $\mathbf{2 0 1 4}$ & $\mathbf{2 0 1 5}$ \\
\hline Deza & M & 35,8 & 35,7 & 35,5 & 31,1 & 33,9 & 34,5 \\
\hline Deza & $\mathrm{V}$ & 64,2 & 64,3 & 64,5 & 68,2 & 66,1 & 65,5 \\
\hline Salnés & $\mathrm{M}$ & 31,5 & 42,9 & 43,5 & 33,1 & 34,6 & 26,5 \\
\hline Salnés & $\mathrm{V}$ & 68,5 & 57,1 & 56,6 & 66,5 & 65,4 & 73,5 \\
\hline Bergantiños & $\mathrm{M}$ & & & & & & 38,8 \\
\hline Bergantiños & $\mathrm{V}$ & & & & & 61,2 \\
\hline
\end{tabular}




\begin{tabular}{|c|c|c|c|c|c|c|}
\hline \multicolumn{7}{|c|}{ Indicador C.6 Edad de acceso } \\
\hline \multicolumn{7}{|c|}{ Porcentaje de niños/as atendidos según la edad en la presentación de la solicitud } \\
\hline UDIAF & $0-1$ & $1-2$ & 2.3 & $3-4$ & 4-5 & $5-6$ \\
\hline Deza & 5,3 & 11,9 & 23,6 & 20,3 & 21,1 & 17,8 \\
\hline Salnés & 2,7 & 6,7 & 20,8 & 23,5 & 26,4 & 19,9 \\
\hline Bergantiños & 0 & 5,8 & 19,8 & 18,6 & 34,9 & 20,9 \\
\hline
\end{tabular}

Fuente: elaboración propia.

b. Indicadores estructurales y profesionales: para analizar la condición e idoneidad de las instalaciones y la accesibilidad (Indicadores EI) según la valoración de las familias a través de la correspondiente escala de satisfacción, la adecuación de las unidades a las normas de la administración (E2), la actualización formativa y actividad investigadora de los equipos (E3), la modernización de los soportes de gestión (E4) y la estabilidad laboral de los profesionales ( $\left.\mathrm{E}_{5}\right)$.

Tabla 2. Indicadores estructurales y profesionales

\begin{tabular}{|l|l|c|c|c|c|c|c|}
\hline Indicador E.1.1 Instalaciones \\
\hline Estado de las instalaciones de las UDIAF valorado por las familias* \\
\hline UDIAF & $\mathbf{2 0 1 0}$ & $\mathbf{2 0 1 1}$ & $\mathbf{2 0 1 2}$ & $\mathbf{2 0 1 3}$ & $\mathbf{2 0 1 4}$ & $\mathbf{2 0 1 5}$ \\
\hline Deza & 4,5 & 4,3 & 4,44 & 4,43 & 4,57 & 4,57 \\
\hline Salnés & 3,6 & 3,5 & 3,69 & 3,45 & 3,71 & 3,45 \\
\hline Bergantiños & & & & & & 4,57 \\
\hline${ }^{*}$ Escala 1 a 5
\end{tabular}

\begin{tabular}{|c|c|c|c|c|c|c|}
\hline \multicolumn{7}{|c|}{ Indicador E.1.2 Accesibilidad } \\
\hline \multicolumn{7}{|c|}{ Accesibilidad de las UDIAF valorada por las familias ${ }^{*}$} \\
\hline UDIAF & 2010 & 2011 & 2012 & 2013 & 2014 & 2015 \\
\hline Deza & 4,5 & 4,1 & 4,47 & 4,49 & 4,73 & 4,68 \\
\hline Salnés & 4,1 & 3,9 & 4,4 & 4,25 & 4,37 & 4,36 \\
\hline Bergantiños & & & & & & 4,32 \\
\hline
\end{tabular}

\begin{tabular}{|l|l|c|c|c|c|c|c|}
\hline Indicador E.2 Adecuación normativa \\
\hline Control de la adecuación de las UDIAF a las normativas existentes \\
\hline UDIAF & $\mathbf{2 0 1 0}$ & $\mathbf{2 0 1 1}$ & $\mathbf{2 0 1 2}$ & $\mathbf{2 0 1 3}$ & $\mathbf{2 0 1 4}$ & $\mathbf{2 0 1 5}$ \\
\hline DEZA & & & & & & \\
\hline Autorización RUEPSS & & & & & $\mathrm{x}$ & $\mathrm{x}$ \\
\hline Acreditación Decreto & & & & & $\mathrm{pte}$ & $\mathrm{pte}$ \\
\hline Centro sanitario & & & & & $\mathrm{x}$ & $\mathrm{x}$ \\
\hline Cons. informado & $\mathrm{x}$ & $\mathrm{x}$ & $\mathrm{x}$ & $\mathrm{x}$ & $\mathrm{x}$ & $\mathrm{x}$ \\
\hline Protección datos & $\mathrm{x}$ & $\mathrm{x}$ & $\mathrm{x}$ & $\mathrm{x}$ & $\mathrm{x}$ & $\mathrm{x}$ \\
\hline Contrato asistencial & & & & & $\mathrm{x}$ & $\mathrm{x}$ \\
\hline SALNES & & & & & & \\
\hline Autorización RUEPSS & & & & & $\mathrm{x}$ & $\mathrm{x}$ \\
\hline Acreditación Decreto & & & & & $\mathrm{pte}$ & $\mathrm{pte}$ \\
\hline Centro sanitario & & & & & $\mathrm{x}$ & $\mathrm{x}$ \\
\hline Cons. informado & $\mathrm{x}$ & $\mathrm{x}$ & $\mathrm{x}$ & $\mathrm{x}$ & $\mathrm{x}$ & $\mathrm{x}$ \\
\hline Protección datos & $\mathrm{x}$ & $\mathrm{x}$ & $\mathrm{x}$ & $\mathrm{x}$ & $\mathrm{x}$ & $\mathrm{x}$ \\
\hline Contrato asistencial & & & & & $\mathrm{x}$ & $\mathrm{x}$ \\
\hline
\end{tabular}




\begin{tabular}{|l|c|c|c|c|c|c|}
\hline BERGANTIÑOS & & & & & & \\
\hline Autorización RUEPSS & & & & & $\mathrm{x}$ & $\mathrm{x}$ \\
\hline Acreditación Decreto & & & & & $\mathrm{pte}$ & $\mathrm{pte}$ \\
\hline Centro sanitario & & & & & $\mathrm{x}$ & $\mathrm{x}$ \\
\hline Cons. informado & & & & & $\mathrm{x}$ & $\mathrm{x}$ \\
\hline Protección datos & & & & & $\mathrm{x}$ & $\mathrm{x}$ \\
\hline Contrato asistencial & & & & & $\mathrm{x}$ & $\mathrm{x}$ \\
\hline
\end{tabular}

\begin{tabular}{|c|c|c|c|c|c|c|}
\hline \multicolumn{7}{|c|}{ Indicador E.3.1 Formación } \\
\hline \multicolumn{7}{|c|}{$\begin{array}{l}\text { Horas de formación dedicadas por los equipos a actividades formativas organizadas por la dirección de las } \\
\text { UDIAF }\end{array}$} \\
\hline UDIAF & 2010 & 2011 & 2012 & 2013 & 2014 & 2015 \\
\hline \multicolumn{7}{|l|}{ DEZA } \\
\hline Gestión de procesos & 16 & 24 & 31 & 20 & 8 & 16 \\
\hline Formación continua & 40 & 8 & 27 & 18 & 8 & 13 \\
\hline \multicolumn{7}{|l|}{ SALNES } \\
\hline Gestión de procesos & 16 & 24 & 31 & 20 & 8 & 16 \\
\hline Formación continua & 40 & 8 & 27 & 10 & 8 & 13 \\
\hline \multicolumn{7}{|l|}{ BERGANTIÑOS } \\
\hline Gestión de procesos & & & & & 30 & 24 \\
\hline Formación continua & & & & & 5 & 40 \\
\hline
\end{tabular}

\section{Indicador E.3.2 Estudios e investigaciones}

Control de la participación de las UDIAF en actividades de estudio e investigación

\begin{tabular}{|l|c|c|c|c|c|c|}
\hline UDIAF & 2010 & 2011 & 2012 & 2013 & 2014 & 2015 \\
\hline Deza & $\mathrm{x}$ & $\mathrm{x}$ & $\mathrm{x}$ & $\mathrm{x}$ & $\mathrm{x}$ & $\mathrm{x}$ \\
\hline Salnés & $\mathrm{x}$ & $\mathrm{x}$ & $\mathrm{x}$ & $\mathrm{x}$ & $\mathrm{x}$ & $\mathrm{x}$ \\
\hline Bergantiños & & & & & & $\mathrm{x}$ \\
\hline
\end{tabular}

Indicador E.4. Adecuación de soportes

Control de tipo de soportes utilizados en la actividad de las UDIAF

\begin{tabular}{|c|c|c|c|c|c|c|}
\hline UDIAF & 2010 & 2011 & 2012 & 2013 & 2014 & 2015 \\
\hline \multicolumn{7}{|l|}{ DEZA } \\
\hline Papel/Office & $\mathrm{x}$ & & & & & \\
\hline Papel/Aplicación integrada & & $x$ & & & & \\
\hline Aplicación integrada & & & $\mathrm{x}$ & $\mathrm{x}$ & $\mathrm{x}$ & $\mathrm{x}$ \\
\hline \multicolumn{7}{|l|}{ SALNES } \\
\hline Papel/Office & $\mathrm{x}$ & & & & & \\
\hline Papel/Aplicación integrada & & $\mathrm{x}$ & & & & \\
\hline Aplicación integrada & & & $x$ & $x$ & $x$ & $x$ \\
\hline \multicolumn{7}{|l|}{ BERGANTIÑOS } \\
\hline \multicolumn{7}{|l|}{ Papel/Office } \\
\hline \multicolumn{7}{|l|}{ Papel/Aplicación integrada } \\
\hline Aplicación integrada & & & & & & $\mathrm{x}$ \\
\hline
\end{tabular}

Indicador E.5. Estabilidad profesional.

Porcentaje de profesionales con contrato indefinido

\begin{tabular}{|l|c|c|c|c|c|c|}
\hline Porcentaje de profesionales con contrato indefinido & $\mathbf{2 0 1 0}$ & $\mathbf{2 0 1 1}$ & $\mathbf{2 0 1 2}$ & $\mathbf{2 0 1 3}$ & $\mathbf{2 0 1 4}$ & $\mathbf{2 0 1 5}$ \\
\hline UDIAF & $0 \%$ & $75 \%$ & $75 \%$ & $100 \%$ & $100 \%$ & $100 \%$ \\
\hline Salnés (4) & $0 \%$ & $75 \%$ & $75 \%$ & $100 \%$ & $100 \%$ & $100 \%$ \\
\hline Bergantiños (4) & & & & & $25 \%$ & $25 \%$ \\
\hline Entre paréntesis, $n^{\circ}$ de profesionales
\end{tabular}

Entre paréntesis, $\mathrm{n}^{\circ}$ de profesionales

Fuente: elaboración propia. 
c. Indicadores de actividad: al objeto de ofrecer datos empíricos sobre las actuaciones realizadas por las unidades de acuerdo a su glosario de prestaciones individuales y comunitarias ${ }^{3}$. Las prestaciones individuales (AI) incluyen las consultas de acogida (A2), las sesiones diagnósticas $\left(\mathrm{A}_{3}\right)$, las sesiones de apoyo a la familia y al niño (A4) y las sesiones de orientación $\left(\mathrm{A}_{5}\right)$. En las prestaciones comunitarias (A8) se incluyen, entre otras, las actuaciones de coordinación (A6) y las reuniones de equipo $\left(\mathrm{A}_{7}\right)$ como indicadores elegidos para seguir el trabajo interdisciplinario externo e interno del equipo.

Tabla 3. Indicadores de actividad
\begin{tabular}{|l|c|c|c|c|c|c|}
\hline Indicador A.1: Prestaciones individuales \\
\hline No total de prestaciones individuales/año \\
\hline UDIAF & 2010 & $\mathbf{2 0 1 1}$ & $\mathbf{2 0 1 2}$ & $\mathbf{2 0 1 3}$ & $\mathbf{2 0 1 4}$ & $\mathbf{2 0 1 5}$ \\
\hline Deza & 1869 & 2355 & 2235 & 2200 & 2045 & 1940 \\
\hline Salnés & 2481 & 2409 & 2594 & 2792 & 2780 & 2484 \\
\hline Bergantiños & & & & & 134 & 1422 \\
\hline
\end{tabular}

\begin{tabular}{|c|c|c|c|c|c|c|}
\hline \multicolumn{7}{|c|}{ Indicador A.2: Consultas de acogida } \\
\hline \multicolumn{7}{|c|}{$\mathrm{N}^{\circ}$ de consultas de acogida/año } \\
\hline UDIAF & 2010 & 2011 & 2012 & 2013 & 2014 & 2015 \\
\hline Deza & 53 & 82 & 86 & 115 & 96 & 104 \\
\hline Salnés & 92 & 120 & 68 & 110 & 108 & 98 \\
\hline Bergantiños & & & & & 58 & 136 \\
\hline
\end{tabular}

\begin{tabular}{|c|c|c|c|c|c|c|}
\hline \multicolumn{7}{|c|}{ Indicador A.3: Sesiones diagnósticas } \\
\hline \multicolumn{7}{|c|}{$N^{\circ}$ de sesiones diagnósticas/año } \\
\hline UDIAF & 2010 & 2011 & 2012 & 2013 & 2014 & 2015 \\
\hline Deza & 83 & 118 & 141 & 152 & 136 & 151 \\
\hline Salnés & 269 & 106 & 145 & 182 & 144 & 118 \\
\hline Bergantiños & & & & & 58 & 131 \\
\hline
\end{tabular}

\begin{tabular}{|c|c|c|c|c|c|c|}
\hline \multicolumn{7}{|c|}{ Indicador A.4: Sesiones de apoyo } \\
\hline \multicolumn{7}{|c|}{$N^{\circ}$ de sesiones de apoyo/año } \\
\hline UDIAF & 2010 & 2011 & 2012 & 2013 & 2014 & 2015 \\
\hline Deza & 1440 & 1973 & 1949 & 1906 & 1778 & 1623 \\
\hline Salnés & 1907 & 1922 & 2180 & 2380 & 2375 & 2151 \\
\hline Bergantiños & & & & & 10 & 1026 \\
\hline
\end{tabular}

3. El glosario con el tipo de actuaciones y servicios que prestan las UDIAF puede consultarse en el Anexo I. 


\begin{tabular}{|l|}
\hline Indicador A.5 Sesiones de orientación \\
\begin{tabular}{|l|c|c|c|c|c|c|}
\hline No de sesiones de orientación/año \\
\hline UDIAF & $\mathbf{2 0 1 0}$ & $\mathbf{2 0 1 1}$ & $\mathbf{2 0 1 2}$ & $\mathbf{2 0 1 3}$ & $\mathbf{2 0 1 4}$ & $\mathbf{2 0 1 5}$ \\
\hline Deza & 67 & 14 & 42 & 27 & 30 & 35 \\
\hline Salnés & 13 & 93 & 109 & 105 & 129 & 117 \\
\hline Bergantiños & & & & & 3 & 157 \\
\hline
\end{tabular}
\end{tabular}

\begin{tabular}{|c|c|c|c|c|c|c|}
\hline \multicolumn{7}{|c|}{ Indicador A.6: Actuaciones de coordinación } \\
\hline \multicolumn{7}{|c|}{$N^{\circ}$ de actuaciones de coordinación/año* } \\
\hline UDIAF & 2010 & 2011 & 2012 & 2013 & 2014 & 2015 \\
\hline Deza & 188 & 242 & 259 & 313 & 251 & 257 \\
\hline Salnés & 142 & 167 & 193 & 187 & 212 & 262 \\
\hline Bergantiños & & & & & 136 & 261 \\
\hline
\end{tabular}

\begin{tabular}{|c|c|c|c|c|c|c|}
\hline \multicolumn{7}{|c|}{ Indicador A.7: Sesiones de equipo } \\
\hline \multicolumn{7}{|c|}{$N^{\circ}$ de sesiones de equipo/mes } \\
\hline UDIAF & 2010 & 2011 & 2012 & 2013 & 2014 & 2015 \\
\hline Deza & 7,8 & 7,8 & 8 & 7,6 & 9 & 7,8 \\
\hline Salnés & 7,7 & 7,7 & 7 & 8 & 7,25 & 7,33 \\
\hline Bergantiños & & & & & --.- & 6.41 \\
\hline
\end{tabular}

\begin{tabular}{|l}
\hline Indicador A.8: $\mathbf{N}^{\circ}$ de acciones comunitarias/mes \\
\begin{tabular}{|l|c|c|c|c|c|c|}
\hline$N^{\circ}$ de prestaciones comunitarias/mes \\
\hline UDIAF & $\mathbf{2 0 1 0}$ & $\mathbf{2 0 1 1}$ & $\mathbf{2 0 1 2}$ & $\mathbf{2 0 1 3}$ & $\mathbf{2 0 1 4}$ & $\mathbf{2 0 1 5}$ \\
\hline Deza & 2,9 & 12,53 & 17 & 26 & 20,7 & 21,41 \\
\hline Salnés & 5,9 & 5,9 & 14 & 15,5 & 19,6 & 21,83 \\
\hline Bergantiños & & & & & --- & 25,91 \\
\hline
\end{tabular}
\end{tabular}

Fuente: elaboración propia.

d. Indicadores de procesos: que señalan el nivel de desempeño de las líneas de actuación que se consideran estratégicas para avanzar en la calidad de la asistencia desde la entrada hasta la salida de cada caso: el trabajo en equipo ( $\mathrm{P}_{2}$ y $\left.\mathrm{P}_{3}\right)$, la participación de la familia $\left(\mathrm{P}_{4}, \mathrm{P}_{7}\right.$,
$\mathrm{P}_{9}$ ), el rigor asistencial ( $\mathrm{P}_{1}, \mathrm{P}_{5}, \mathrm{P} 6, \mathrm{P}_{7}$, P8, $\left.\mathrm{P}_{9}, \mathrm{P}_{\mathbf{1}}\right)$. La evaluación la realiza el coordinador externo de las UDIAF siguiendo un esquema de calificación tipo Likert en el que I señala que el procedimiento se realiza de forma muy irregular y 5 de forma muy regular. 
Tabla 4. Indicadores de procesos ${ }^{4}$

P.1 Criterios de elegibilidad. La unidad utiliza criterios de elegibilidad para el acceso a los servicios ${ }^{2}$

Deza 5 Salnés $5 \quad$ Bergantiños 5

P.2 Coordinador de caso. El equipo examina las solicitudes y asigna el coordinador de caso en la sesión de presentación de casos

Deza 5 Salnés $3 \quad$ Bergantiños 4

P.3 Interdisciplinariedad. El equipo estudia y evalúa casos de forma regular una vez a la semana

Deza 4 Salnés $4 \quad$ Bergantiños 3

P.4 Información y orientación. Las familias reciben información y orientación sobre los recursos y prestaciones públicas disponibles en las redes comunitarias

Deza 4 Salnés 4 Bergantiños 4

P.5 Consulta de acogida. Todos los expedientes en intervención tienen registrada la consulta de acogida y sus epígrafes básicos de historia de caso
Deza 4
Salnés 4
Bergantiños 5

P.6 Cribado. En las preocupaciones por retraso evolutivo se aplica y se registra siempre una prueba estandarizada de cribado

Deza 4 Salnés $4 \quad$ Bergantiños 4

P.7 Plan personalizado de intervención. Todos los expedientes en intervención cuentan con un registro de procesos y plan personalizado de intervención

Deza 2

Salnés 2

Bergantiños 2

P.8 Revisión. Todos los expedientes en intervención son revisados cada seis meses por el equipo

Deza 2 Salnés $2 \quad$ Bergantiños 2

P.9 Informe de alta. Todos los expedientes en el momento de alta cuentan con informe de alta disponible a petición de la familia

Deza 4 Salnés $4 \quad$ Bergantiños 4

P.10 Registro diagnóstico. Todos los expedientes en el momento de alta cuentan con registro de su perfil diagnóstico según la ODAT

Deza 5 Salnés $5 \quad$ Bergantiños 5

Fuente: elaboración propia.

4. Evaluación por coordinador externo referida a 2015 . 
e. Indicadores de resultados: para facilitar la evaluación de los objetivos asistenciales de las unidades y la calidad de los servicios se controla: el número de familias asistidas (RI), los nuevos ingresos por cada UDIAF $\left(\mathrm{R}_{5}\right)$ y la rotación anual (R.6), el origen de las derivaciones $\left(\mathrm{R}_{2}\right)$, la demora en la acogida $\left(\mathrm{R}_{3}\right)$ y la existencia o no de lista de espera $\left(\mathrm{R}_{4}\right)$. Finalmente, se registran dos indicadores a través de los cuales se obtiene la satisfacción de los usuarios por los servicios prestados, su fidelización $\left(\mathrm{R}_{7}\right)$ y su valoración de los servicios recibidos (R8)

\section{Tabla 5. Indicadores de resultados}

\section{Indicador R.1: Niños/familias 0-6 atendidas}

$N^{\circ}$ de niños/familias atendidos/año

\begin{tabular}{|l|c|c|c|c|c|c|}
\hline UDIAF & $\mathbf{2 0 1 0}$ & $\mathbf{2 0 1 1}$ & $\mathbf{2 0 1 2}$ & $\mathbf{2 0 1 3}$ & $\mathbf{2 0 1 4}$ & $\mathbf{2 0 1 5}$ \\
\hline Deza & 81 & 112 & 110 & 132 & 122 & 110 \\
\hline Salnés & 127 & 133 & 124 & 121 & 128 & 118 \\
\hline Bergantiños & & & & & 23 & 85 \\
\hline
\end{tabular}

Indicador R.2: Detección/Derivación

Porcentaje de niños/as atendidos según procedencia

\begin{tabular}{|l|c|c|c|}
\hline Detección/Derivación & Deza & Salnés & Bergantiños \\
\hline Comunidad & 31,1 & 26,7 & 18,6 \\
\hline S. Educativos $2^{\circ}$ ciclo & 16,7 & 28,6 & 37,2 \\
\hline S. Educativos $1^{\circ}$ ciclo & 6,6 & 10,2 & 4,7 \\
\hline S. de Salud & 37,0 & 27,0 & 37,2 \\
\hline S. Sociales & 8,2 & 7,3 & 2,3 \\
\hline Total & 100,0 & 100,0 & 100,0 \\
\hline
\end{tabular}

Casos: 257 Deza, 371 Salnés, 86 Bergantiños

\begin{tabular}{|l|l|c|c|c|c|c|c|}
\hline Indicador R.3: Demora \\
\hline Demora media entre fecha de entrada y fecha de acogida en días \\
\hline UDIAF & $\mathbf{2 0 1 0}$ & $\mathbf{2 0 1 1}$ & $\mathbf{2 0 1 2}$ & $\mathbf{2 0 1 3}$ & $\mathbf{2 0 1 4}$ & $\mathbf{2 0 1 5}$ \\
\hline Deza & -- & 13,9 & 12,4 & 12,7 & 19,7 & 20,7 \\
\hline Salnés & & 24,0 & 21,0 & 20,0 & 18,6 & 26,2 \\
\hline Bergantiños & & & & & & 29,0 \\
\hline
\end{tabular}

\begin{tabular}{|l|l|l|c|c|c|c|c|}
\hline Indicador R.4: Lista de espera \\
\hline No de niños/as en lista de espera a 31 diciembre & $\mathbf{2 0 1 0}$ & $\mathbf{2 0 1 1}$ & $\mathbf{2 0 1 2}$ & $\mathbf{2 0 1 3}$ & $\mathbf{2 0 1 4}$ & $\mathbf{2 0 1 5}$ \\
\hline UDIAF & & 20 & 33 & 20 & 1 & 1 \\
\hline Deza & & 32 & 1 & 11 & 6 & 7 \\
\hline Salnés & & & & & & 7 \\
\hline Bergantiños
\end{tabular}




\begin{tabular}{|c|c|c|c|c|c|c|}
\hline \multicolumn{7}{|c|}{ Indicador R.5: Ingresos } \\
\hline \multicolumn{7}{|c|}{$N^{\circ}$ de ingresos /año } \\
\hline UDIAF & 2010 & 2011 & 2012 & 2013 & 2014 & 2015 \\
\hline Deza & -- & 39 & 42 & 56 & 44 & 54 \\
\hline Salnés & -- & 65 & 43 & 55 & 54 & 49 \\
\hline Bergantiños & & & & & 28 & 63 \\
\hline
\end{tabular}

\begin{tabular}{|l}
\begin{tabular}{|l|c|c|c|c|c|c|}
\hline Indicador R.6: Rotación. \\
\hline Relación casos atendidos/ingresos (porcentaje) \\
\hline UDIAF & $\mathbf{2 0 1 0}$ & $\mathbf{2 0 1 1}$ & $\mathbf{2 0 1 2}$ & $\mathbf{2 0 1 3}$ & $\mathbf{2 0 1 4}$ & $\mathbf{2 0 1 5}$ \\
\hline Deza & & 34,8 & 38,18 & 42,42 & 36,00 & 49,09 \\
\hline Salnés & & 48,8 & 34,67 & 45,45 & 42,18 & 41,50 \\
\hline Bergantiños & & & & & 100 & 76,47 \\
\hline
\end{tabular}
\end{tabular}

\section{Indicador R.7: Fidelización}

Relación de altas voluntarias/familias atendidas (porcentaje)

\begin{tabular}{|l|c|c|c|c|c|c|}
\hline UDIAF & $\mathbf{2 0 1 0}$ & $\mathbf{2 0 1 1}$ & $\mathbf{2 0 1 2}$ & $\mathbf{2 0 1 3}$ & $\mathbf{2 0 1 4}$ & $\mathbf{2 0 1 5}$ \\
\hline Deza & -- & 4,5 & 2,7 & 3,03 & 4,1 & 0 \\
\hline Salnés & -- & 3 & 1,6 & 3,3 & 3,9 & 0 \\
\hline Bergantiños & & & & & 0 & 1,1 \\
\hline
\end{tabular}

Indicador R.8: Satisfacción de las familias

Evaluación por las familias de los servicios de las UDIAFS

\begin{tabular}{|l|c|c|c|c|c|c|}
\hline UDIAF & $\mathbf{2 0 1 0}$ & $\mathbf{2 0 1 1}$ & $\mathbf{2 0 1 2}$ & $\mathbf{2 0 1 3}$ & $\mathbf{2 0 1 4}$ & $\mathbf{2 0 1 5}$ \\
\hline Deza & 4,6 & 4,4 & 4,74 & 4,68 & 4,76 & 4,81 \\
\hline Salnés & 4,6 & 4,4 & 4,75 & 4,8 & 4,88 & 4,79 \\
\hline Bergantiños & & & & & & 4,72 \\
\hline Escala 1 a 5 & & & &
\end{tabular}

Fuente: elaboración propia.

Los resultados obtenidos por las unidades se han evaluado anualmente en sesiones de trabajo de cada UDIAF, pero también en sesiones conjuntas (de todas las UDIAF), siendo estas últimas habitualmente dos sesiones anuales de ocho horas de duración, todos los años desde 2009 a 2015 , con documentación previa. La primera de las sesiones conjuntas se dedica a la evaluación cuantitativa y cualitativa de los datos anuales mientras que la segunda se centra en la evaluación de procesos apoyada en el estudio de casos.
Posteriormente, en febrero de 2016 se ha realizado una valoración del conjunto del período (2009-20I6), cuyo resultado global se expone en el informe técnico ya citado, UDIAFS. Servizos comunitarios de Atención Temperá (Ponte et al., 2016) y uno de cuyos aspectos lo constituye la discusión y la calificación de cada indicador por los profesionales que es parte central de este artículo. 
La calificación de los indicadores se realizó mediante una escala tipo Likert de I a 5 desde "muy poco adecuados" que correspondería a la puntuación I hasta la puntuación 5 que significaría "muy adecuados", con el fin de seleccionar aquellos que los profesionales propondrían para formar parte de un "cuadro de mando" o "scoreboard" integral que en su día pueda servir para establecer un procedimiento de acreditación, seguimiento y auditoría de los servicios. Para formar parte de la selección final se estableció una nota de corte mínima de 4 puntos ("bastante adecuado").

\section{Resultados}

Los indicadores que superan la nota de corte y obtienen mayor puntuación son los siguientes:

- Indicadores de alcance

C. 6 Edad de acceso: 4,8

C.I Porcentaje de población o-6 atendida: 4,7

- Indicadores estructurales y profesionales E.2 Adecuación normativa: 4,5

E.I Accesibilidad: 4,4

E. 5 Estabilidad profesional: 4,4

- Indicadores de actividad

A. $6 \mathrm{~N}^{\circ}$ de actuaciones de coordinación: 4,5 A.I $\mathrm{N}^{0}$ de prestaciones individuales: 4,2

A. $4 \mathrm{~N}^{\circ}$ de sesiones de apoyo: 4,2

- Indicadores de procesos

P.4 Información y orientación: 5, 0

P. 2 Coordinador de caso: 4,7

P.9 Informe de alta: 4,7

P. 5 Consulta de acogida: 4,5

P.6 Cribado: 4,5

P. Io Registro diagnóstico: 4,4

- Indicadores de resultados

R. 8 Satisfacción de las familias: 5,0

R.I Niños/familias atendidas/año: 4,8

R. 5 Ingresos: 4,4

\author{
R.2 Detección/Derivación: 4,2 \\ R.3 Demora: 4,2
}

Los profesionales han considerado conveniente incorporar un nuevo indicador de alcance, utilizando el registro mensual de las unidades, que da cuenta de la distribución territorial por ayuntamientos de las solicitudes recibidas. Este nuevo indicador es el siguiente:

C.7 Distribución territorial por ayuntamiento. Distribución porcentual de los niños/as atendidos/as por ayuntamiento de residencia

\begin{tabular}{|c|c|c|}
\hline $\mathbf{N}^{\circ}$ & $\begin{array}{l}\text { Nombre y definición del } \\
\text { indicador }\end{array}$ & Puntuación \\
\hline 1 & P.4 Información y orientación & 5 \\
\hline 2 & R.8 Satisfacción de las familias & 5 \\
\hline 3 & C.6 Edad de acceso & 4,8 \\
\hline 4 & $\begin{array}{l}\text { R.1 Niños/familias atendidas/ } \\
\text { año }\end{array}$ & 4,8 \\
\hline 5 & $\begin{array}{l}\text { C.1 Porcentaje de población 0-6 } \\
\text { atendida }\end{array}$ & 4,7 \\
\hline 6 & P.2 Coordinador de caso & 4,7 \\
\hline 7 & P.9 Informe de alta & 4,7 \\
\hline 8 & E.2 Adecuación normativa & 4,5 \\
\hline 9 & $\begin{array}{l}\text { A. } 6 \mathrm{~N}^{\circ} \text { de actuaciones de } \\
\text { coordinación }\end{array}$ & 4,5 \\
\hline 10 & P.5 Consulta de acogida & 4,5 \\
\hline 11 & P.6 Cribado & 4,5 \\
\hline 12 & E.1 Accesibilidad & 4,4 \\
\hline 13 & E.5 Estabilidad profesional & 4,4 \\
\hline 14 & P.10 Registro diagnóstico & 4,4 \\
\hline 15 & R.5 Ingresos & 4,4 \\
\hline 16 & $\begin{array}{l}\text { A. } 1 \mathrm{~N}^{\circ} \text { de prestaciones } \\
\text { individuales }\end{array}$ & 4,2 \\
\hline 17 & A.4 $\mathrm{N}^{\circ}$ de sesiones de apoyo & 4,2 \\
\hline 18 & R.3 Demora & 4,2 \\
\hline 19 & R.2 Detección/Derivación & 4,2 \\
\hline 20 & $\begin{array}{l}\text { C.7 Distribución territorial por } \\
\text { ayuntamiento }\end{array}$ & S.C ${ }^{*}$ \\
\hline
\end{tabular}

*s.c: sin calificar, explicación en el texto.

Fuente: elaboración propia 


\section{Discusión}

Los profesionales de las UDIAF han seleccionado en primer lugar (puntuación $5,0)$ indicadores que tienen que ver con la información y orientación a las familias (P.4) y con su grado de satisfacción respecto a los servicios que reciben (R.8). Esta unanimidad y alta calificación parece confirmar la atribución a las familias del papel central que hoy día se les reconoce en la atención temprana y, en cierto modo, refleja la propia satisfacción de los profesionales al comprobar año tras año la alta consideración que, en su caso, les devuelven las familias en dichas encuestas. Las encuestas de satisfacción que emplean las UDIAF son un resumen de la escala Measure of Process of Care - MPOC (King et al., I995, I996, 2004). La escala pregunta a los padres sobre accesibilidad, estado de instalaciones y horarios (área I), acogida (área 2), posibilidad de hablar de sus preocupaciones (área 3), satisfacción con las informaciones y orientaciones (área 4), coordinación con otros recursos (área 5) y confianza en los profesionales (área 6). La encuesta tiene además un espacio de calificación de la satisfacción global y otros dos para que las familias expongan libremente lo que consideran mejor y peor en la UDIAF. En los últimos siete años la calificación global de las unidades ha progresado hasta niveles muy estimables $(4,79$ Salnés; 4,82 Deza; 4,72 Bergantiños, en 2016) con una participación bastante numerosa de las familias (en torno al 50\%). La escala MPOC se seleccionó para recoger la valoración de la calidad percibida por las familias por ser un instrumento muy difundido y utilizado en diferentes países desde hace años, mostrando una excelente consistencia interna, fiabilidad y validez (Kertoy, M. K. et al., 2013).

En el segundo tramo de puntuación de esta clasificación de indicadores aparecen los que aportan datos sobre el alcance de la unidad en el territorio que se encuentra bajo su responsabilidad. La edad de acceso (C.6: puntuación 4,8), el número de niños/familias atendidos $($ R.I $=4,8)$ y el nivel de cobertura de la población o-6 (C.I = 4,7) son los más valorados y no cabe duda de la importancia que tiene, a efectos de planificación asistencial, conocer si se está llegando a la población diana y poder ajustar la demanda con la estructura y medios del equipo. Para servicios territorializados, como son los servicios de atención temprana, el dato de cobertura de la población diana resulta primordial pues es un testigo bastante fiable del cumplimiento cuantitativo de los objetivos del servicio. Como puede comprobarse, cada UDIAF, con un equipo de tres profesionales, puede atender una media de alrededor de Ioo familias/ año, lo que traducido a cobertura de población 0-6 se sitúa en el entorno del $2 \%$ en la comarca del Salnés (I00.000 h.) y casi llega al $6 \%$ en la comarca del Deza (45.000 h.) Lo que también nos señala que nuestra realidad no está lejos de otras comunidades autónomas, como la propia Cataluña (población diana 5,5\%) cuya Red está implantada desde hace años. $\mathrm{O}$ de otros países como EE. UU, donde los servicios de atención temprana llegan casi al $3 \%$ de la población infantil de o a 3 años (ECTA, 20I6). En cuanto a la edad de entrada en las UDIAF, la tasa de solicitudes entre o y 2 años es todavía claramente inferior a la registrada en otras redes $(28 \%$ Cataluña, frente a un I I \% global en las UDIAF) lo que señala con claridad una dirección de mejora. Llama la atención también que se hayan descartado como menos útiles los indicadores que permiten conocer el tipo de problemas que acceden a las unidades (C.2, tipología de la demanda) o su perfil diagnóstico según la ODAT (C.3), aunque posteriormente se considere importante garantizar, en el nivel metodológico, que todos los casos en el momento del alta cuenten con el registro de dicho perfil (P.ıo). Aparentemente este resultado es consecuencia de una disconformidad de los profesionales con algunos aspectos que propone la ODAT, como la dificultad para graduar y cualificar sus categorías, lo que "les obligaría" a "elevar" todos los problemas evolutivos al nivel de "trastornos del desarrollo", con el consiguiente efecto de incluir al niño en una categoría patológica, sin más matices. Pero cabe decir, por nuestra parte, que la distribución tipológica de la demanda y el mayor o menor peso de unos tipos diagnósticos nos parece imprescindible para conformar los 
dispositivos asistenciales y profundizar en los perfiles profesionales. De hecho, la ampliación de horas de las plazas de logopedia que se ha dado en las UDIAF a lo largo de los últimos siete años tiene mucho que ver con haber podido mostrar a la administración pública que uno de cada tres niños que acuden a las unidades presenta algún problema de lenguaje o comunicación (Ponte, 20I5).

En un tercer tramo, puede subrayarse la buena puntuación obtenida por determinados procesos, como la asignación en equipo del coordinador de caso $(\mathrm{P} .2=4,7)$, el rigor en la consulta de acogida $(\mathrm{P} .5=4,5)$, el uso estandarizado de pruebas de cribado (P.6 = $4,5)$ o la sistematicidad de los informes de alta $($ P.9 = 4,7), mientras que otros como el plan personalizado de intervención (P.7) o la regularidad en las revisiones (P.8) no alcanzan la línea de corte. Hasta cierto punto se obtiene un resultado coherente con la mayor o menor asimilación de los procedimientos que implican algunas tareas transdisciplinarias que implica el trabajo como coordinador de caso (Care Coordination Network, 2008; Bruder, 2010; Forster y Webster, 20I2). No es fácil cambiar formatos previos de anamnesis, observación, interpretación y, sobre todo, de relación con las familias y con otros servicios. Los profesionales reconocen dificultades para incorporar en su hacer diario la perspectiva de las familias y lograr su cooperación activa, lo que podría ser una de las razones para explicar los resultados. Aunque se defienda, como hemos visto antes, el papel central de las familias, se reconoce como un área de especial complejidad la “relación con las familias” y, en general, el encuentro de fórmulas idóneas para hablar, acordar, mantener alianzas y recoger otras posiciones en la intervención. En la práctica se aprecian las dificultades de sobreponerse al modelo prescriptivo, de pautas, indicaciones, etc., mientras que todavía hay poca experiencia en la confección de programas conjuntos de actuación. Los programas personalizados de intervención exigen un proceso más laborioso que la dinámica tradicional de diagnóstico y tratamiento. Requieren competencias transversales en el desarrollo infantil, flexibilidad para establecer compromisos con cada familia y con sus entornos, cada uno distinto, y una amplia experiencia de la vida cotidiana de los niños y de sus cuidadores, de las prácticas de crianza y de las actividades que se desarrollan en las escuelas infantiles. La práctica del llamado 'partnership', del trabajo en equipo y de la coordinación, sigue siendo un área en el que se reclama continuamente más formación.

En esta selección que surge de la calificación profesional se ubican a continuación un grupo diverso de indicadores cuantitativos. Entre ellos, el tiempo de demora en la acogida (R.3) un indicador inexcusable en los programas de intervención temprana de todo el mundo y que, en el caso de las UDIAF, se sitúa entre los 20 y 30 días, un promedio aceptable teniendo en cuenta que no hay lista de espera ni de urgencia. En cuanto a los datos empíricos de actividad es apreciable que el indicador mejor puntuado sea el de las actividades de coordinación en el trabajo de caso $($ A. $6=4,5)$ lo que apunta a la preocupación por significar y controlar el tiempo que se dedica a colaborar con otros servicios comunitarios; una preocupación estimable si tenemos en cuenta las dificultades que suele entrañar salir del territorio propio (mi sala, mi disciplina, mi interpretación, mi centro...) y una preocupación que también se reflejaría en querer conocer en cada momento donde se origina la demanda. En este sentido la propuesta de un nuevo indicador C.7 que informe del lugar de residencia de los solicitantes provendría del interés por disponer de feedback respecto a la implantación de la UDIAF en las distintas zonas del territorio. Sin embargo, sorprende el descarte de los indicadores de actividad que dan cuenta del $\mathrm{n}^{\circ}$ de reuniones de equipo (A.7) o del $\mathrm{n}^{\circ}$ de coordinaciones institucionales (A.8), algo que los interesados explican por una aparente obviedad de las referidas actividades, desde la asunción de que el trabajo en equipo o de coordinación externa es consustancial con la atención temprana y por lo tanto podría mantenerse invisible o al margen de un seguimiento. Sin embargo, las referencias que se comunican de manera informal desde otras comunidades autónomas o que se publican en otros países (Encuesta FranzL, Baviera, 20I0) 
nos dice que existen grandes diferencias en el $\mathrm{n}^{\mathrm{o}}$ de sesiones de equipo que se realizan, en su periodicidad, en su duración, en sus contenidos y en su organización, por lo cual no está de más conocer cómo se desarrolla el trabajo de equipo. En el caso de las UDIAF se trata de una actividad principal. Los equipos se reúnen habitualmente dos veces por semana, una o dos horas por sesión y analizan de forma regular las entradas y el curso de los casos en intervención, pero el contacto y el intercambio de información es continuo, facilitado por la dimensión del equipo. De cualquier manera, los interesados han preferido elegir las acciones vinculadas directamente al trabajo de caso: $\mathrm{n}^{\circ}$ de coordinaciones (A.6), $\mathrm{n}^{\mathrm{o}}$ de sesiones de apoyo (A.4) y suma de prestaciones individuales (A.I).

Por último, salpicados aquí y allá, aparecen indicadores que reclaman vigilancia sobre aspectos estructurales como la adecuación normativa $($ E.2 $=4.5)$ o la estabilidad profesional $($ E.4=4.4). Al subrayar la adecuación normativa se quiere señalar la importancia de cumplir con requisitos como la protección de datos, el consentimiento informado o la responsabilidad civil. Pero sobretodo se manifiesta el deseo de los profesionales de que exista un contrato asistencial ${ }^{5}$ que regule los aspectos clave de las relaciones entre las familias y los profesionales. Algo semejante parece apuntar la puntuación otorgada a la estabilidad profesional, en la que creemos apreciar, más que una reclamación laboral, la demanda de proteger la inversión formativa y la experiencia práctica obtenida en un equipo de atención temprana. La precariedad, la discontinuidad y el cambio frecuente de profesionales en los equipos sanitarios, sociales o educativos que atienden a niños y familias con necesidades especiales produce mucha desorientación y resulta muy poco económico para los directamente afectados (Soriano, 2005).

5. Debe tenerse en cuenta que el actual contrato asistencial de las UDIAF regula las características del servicio, duración, sistema de admisión, funciones, personal disponible, normas de funcionamiento, régimen económico, derechos, deberes y sistema de baja.
Se trata en fin de una selección que corresponde a la mirada de un pequeño grupo de profesionales trabajando en unidades recién implantadas y en una comunidad autónoma que desde 2015 dispone de un nuevo protocolo asistencial, hechos que indudablemente habrán tenido su cuota de influencia a la hora de puntuar. Somos conscientes de esta carga de circunstancias, de subjetividad y de eventuales sesgos, como no podía ser menos cuando utilizamos indicadores basados en encuestas de satisfacción o valoraciones en las que se es juez y parte, y cuando el $\mathrm{n}^{\mathrm{o}}$ de unidades estudiadas es una muestra pequeña dentro de una red de servicios. Pero también somos conscientes de la importancia de contar con la participación de los usuarios y de los profesionales en la evaluación de las políticas públicas. Por eso, con todas las limitaciones de la experiencia, creemos que se trata de una radiografía que nos permite ir un poco más allá en el análisis de los servicios, en la caracterización de sus procesos o en el juicio sobre sus resultados, que puede ayudar al desarrollo de la Red Gallega de Atención Temprana pero también a otras administraciones y recursos, permitiéndoles contrastar y comparar experiencias y datos, conocer mejor las necesidades y las demandas de las partes, y dar cuenta de aspectos importantes de funcionamiento y rendimiento de un servicio público que se encuentra en plena expansión.

\section{Reflexiones finales}

Además de ser necesaria, sabemos que la atención temprana es una prestación compleja, plagada de variables e influencias $\mathrm{y}$, consecuentemente, difícil de medir, por la riqueza de ámbitos, dimensiones, niveles, teorías, prácticas, recursos, demandas e intereses en juego, y porque la experiencia todavía es muy limitada, muy parcial y muy diferente en unos y en otros lugares, incluso dentro de una misma comunidad autónoma. De esta manera, aunque en su momento haya existido una amplia coincidencia en los principios y en 
los objetivos de la intervención (Libro Blanco, 2000) la realidad actual nos advierte de que se ha producido una gran dispersión normativa, de criterios, de medios y de procedimientos. El derecho de los niños a la atención temprana está prácticamente reconocido en toda España, pero su instrumentación y evaluación es muy diferente produciendo todo tipo de interrogantes a los técnicos de la administración, a las familias o a los profesionales que se interesan por los servicios y se desplazan de una u otra comunidad autónoma e incluso de una u otra ciudad.

Pensamos que todavía hay mucho camino por recorrer hasta encontrar un modelo básico y consensuado de financiación, planificación, organización, coordinación, prestación y evaluación de servicios en el que puedan encontrarse igualmente cómodos las administraciones, los departamentos y organizaciones proveedoras de servicios, los profesionales que los prestan directa e indirectamente, las familias y no digamos los niños/as, destinatarios últimos del esfuerzo social y testigos habitualmente sin voz de la mayor o menor bondad de los servicios.

Rubert (20I0) ha propuesto abrir líneas de trabajo en los niveles micro (recursos y procedimientos), meso (organización del CDIAT), macro (organización interdepartamental de los programas a niños con problemas de desarrollo) y supermacro (políticas públicas de infancia y parentalidad), pero él mismo reconoce las enormes dificultades de esa tarea si no encuentra el respaldo decidido de las administraciones públicas, de las entidades del tercer sector y de los grupos profesionales.

Porque parece indudable que en muchos sectores la evaluación se vive todavía como un riesgo y no como una oportunidad. Venimos de una época en la que, salvo excepciones, ha habido una gran reserva documental, una actitud muy defensiva y de proteccionismo a la hora de que cada profesional explicara lo que hacía en el trabajo de caso, cada equipo expusiera sus datos y esquemas de trabajo o cada administración diera cuenta transparente de sus criterios y cuantías de financiación. Una reserva para la que seguramente podrán encontrarse causas múltiples y razonables (de desconocimiento, inseguridad, falta de experiencia, exceso de demandas, carencias en la sistematización, etc.) pero que, de alguna manera, ha limitado la reflexión sobre la práctica profesional, sobre la dinámica de los equipos, la coordinación interdepartamental o la colaboración con las familias. Y que explica, muy probablemente, una gran parte de las dificultades que existen para unificar financiación, cartera de servicios, ratios, competencias formativas y condiciones laborales.

Habrá que preguntarse si ahora los diferentes actores disponemos ya del bagaje y el grado de madurez para abordar conjuntamente y sin miedos esa tarea, evitando la tentación de reiterar principios, ofrecer atajos o buscar soluciones particulares para ir saliendo del paso; aportando y difundiendo en el espacio público, con todas las limitaciones que tengamos, nuestros datos y nuestros resultados. 
Anexo 1. Glosario de actuaciones

\section{Prestaciones individuales}

- Consulta de acogida: para recibir las demandas personalizadas en las UDIAF. Cada consulta consta como mínimo de dos sesiones, una de acogida de la familia y de sus demandas y otra de observación del niño. Se trata de una intervención clave y de larga duración teniendo en cuenta el tiempo necesario para la escucha y la observación, el análisis y la transcripción. Cada sesión tiene un tiempo medio de dos horas.

- Sesión de diagnóstico: para estudiar, evaluar y orientar las prestaciones personalizadas de las UDIAF. Tiempo medio de una hora incluyendo los registros.

- Sesión de apoyo: para realizar apoyos complejos dentro de las prestaciones personalizadas propias de las UDIAF. Registrar las actuaciones realizadas con el niño y con la familia en sesiones de una hora incluyendo en su caso en estos tiempos la devolución a las familias y los tiempos de transcripción para el registro de evolución y procesos.

- Sesión de orientación o seguimiento: para realizar apoyos sencillos, de asesoramiento, orientación o seguimiento dentro de las prestaciones personalizadas propias de las UDIAF, mediante actuaciones previas o posteriores a la intervención en la propia UDIAF. Tiempo medio de una hora incluyendo los registros.

- Entrevista de coordinación: para realizar entrevistas presenciales de coordinación con otros recursos dentro de las prestaciones personalizados de las UDIAF. Incluyendo también aquellas entrevistas telefónicas, informes y actuaciones que puedan superar los 30 minutos de duración.

- Acción de coordinación: para realizar actividades no presenciales de coordinación con otros recursos dentro de las prestaciones personalizadas de las UDIAF y, en todo caso, de una duración inferior a los treinta minutos.

- Grupo de familias: para conducir las actividades de los grupos de familias dentro de las prestaciones personalizadas de las UDIAF.

- Informes y otras: toda actuación que implica la emisión de información escrita sobre el curso de la intervención en la que se especifican aspectos de valoración o intervención, como por ejemplo informes de devolución, informes para las familias, PPI, altas, etc. Actuaciones de corrección de pruebas, transcripción de sesiones y consultas de acogida, elaboración de materiales, preparación de las sesiones, revisión de casos, elaboración de PPI o revisiones para elaborar informes, cuando precisan de un tiempo suplementario al común.

\section{Prestaciones comunitarias y de calidad}

- Sesión de equipo: sesiones de reunión de equipo. Habitualmente hay dos reuniones formales a la semana de unas dos horas de duración, una con presentación de casos y otra con tareas de evaluación y seguimiento.

- $\quad \mathrm{N}^{\mathrm{o}}$ de casos tratados equipo: $\mathrm{n}^{\mathrm{o}}$ de casos revisados en la reunión del equipo.

- Actividades de formación continua: asistencia de los profesionales a actividades de formación organizadas por las unidades, permisos de formación, evaluaciones, gestión de procesos.

- Coordinación comunitaria: entrevistas y acciones de coordinación general con los recursos comunitarios, sociales, educativos, sanitarios o con los recursos especializados.

- Entrevistas de coordinación: suma de las actuaciones de coordinación de caso y las actuaciones de coordinación comunitaria. 
- Acción de coordinación: suma de las acciones de coordinación de caso y las acciones de coordinación comunitaria.

- Proyección social y comunitaria: actividades globales de sensibilización social.
- Evaluación y coordinación interna: sesiones de evaluación, formación, revisión de casos, acciones de coordinación telefónica con el coordinador externo. 
Referencias bibliográficas

Agencia de Calidad Sanitaria de Andalucía (2012): Manual de estándares de los centros de atención infantil temprana (en línea). <http://www.juntadeandalucia.es/ agenciadecalidadsanitaria/es/certificacion/ centros-y-unidades-sanitarias-/manuales-deestandares-/detalle-de-publicacion/Manualde-estandares-de-los-Centros-de-AtencionInfantil-Temprana.-ME-I2-I_O2/>, acceso I de septiembre de 2016 .

Bruder, M. B. (20I0): "Early childhood intervention: A promise to children and families for their future". Exceptional Children, 76 (3): 339-4I 5 .

Care Coordination Network UK (2008): Key Worker Standards (en línea). <http://www.ccnuk. org.uk/metadot/index.pl?id=2 I 9 I \&isa=Category \&op=show>, acceso Io de Mayo de 2016.

Donabedian, A. (I988): "The quality of care. How can it be assessed?". Journal of the American Medical Association, 260: I743-I748.

Dunst, C. J. (2004): “An integrated framework for practicing early childhood intervention and family support". Perspectives in Education, 22 (2): I-I6.

Early Childhood Technical Center (20I6): Part C. National Program Data (en línea). <http:// ectacenter.org/partc/partcdata.asp $>$, acceso I de septiembre de 2016 .

Encuesta "FranzL (2010): Systemanalyse Interdisziplinäre Frübförderung in Bayern (en línea). <http://www.fruehfoerderung-bayern.de/ projekte/franzl-2oı/>, acceso Io de mayo de 2016.

FEAPS (200I): Atención Temprana. Orientaciones para la calidad. Madrid: Confederación Española de Organizaciones en favor de las Personas con Discapacidad Intelectual.

Forster, J. y Webster, A. (2OI2): The Key Worker: Resources for Early Childhood Intervention Professionals. An Early Childhood Intervention Inclusion (ECII) resource. Malvern, Victoria:
Noah's Ark Inc (en línea). <www.ecii.org.au>, acceso so de mayo de 2016.

García Sánchez, F. A. et al. (2008): "Satisfacción del cliente familia en atención temprana. Valoración de la importancia que otorgan a distintos aspectos del servicio". Siglo Cero: Revista Española sobre Discapacidad Intelectual, 39 (227): 55-74.

GAT (2005): Organización Diagnóstica para la Atención Temprana. Madrid: Real Patronato de Prevención y Atención a Personas con Minusvalía (en línea). <http://riberdis.cedd.net/ handle/I I I 8 I/2978>, acceso I de septiembre de 2016.

GAT (2000): Libro Blanco de Atención Temprana. Madrid: Real Patronato de Prevención y Atención a Personas con Minusvalía.

Generalitat de Catalunya. Proyecto de Orden de acreditación de los Servicios de Atención Precoz y procedimiento de autorización de entidades evaluadoras, Diario Oficial de la Generalitat de Catalunya, I 5 de Julio de 2015, núm. 6913, pp. I.

Giné, C. (2002): La Atenció precoç a Catalunya: serveis i qualitat. Barcelona: ICASS.

Guralnick, M. J. (I997): "Second-generation research in the fieId of early intervention", en Guralnick, M. J. (ed.): The Effectiveness of Early Intervention. Baltimore, Maryland: Paul H. Brookes.

Kertoy, M. K. et al. (2013): “Development of an outcome measurement system for service planning for children and youth with special needs". Child Care Health Dev., 39 (5): $750-$ 759 .

King, S. et al. (2004): "Evaluating health service delivery to children with chronic conditions and their families: development of a refined measure of processes of care (MPOC-20)". Children's Health Care, 33: 35-57.

King, S. et al. (I996): "Parents' perceptions of caregiving: Development and validation of 
a measure of processes". Developmental Medicine and Child Neurology, 38: 757-772.

King, S. et al. (1995): The Measure of Processes of Care (MPOC). A means to assess familycentred behaviours of health care providers. Hamilton, Ontario: McMaster University, Neurodevelopmental Clinical Research Unit.

Moore, T. G. (2008): Supporting young children and their families: Why we need to rethink services and policies. CCCH Working Paper No. I. Parkville, Victoria: Centre for Community Child Health, Royal Children's Hospital (en línea). <http://www.rch.org.au/ emplibrary/ccch/Need_for_change_working_ paper.pdf>, acceso I de septiembre de 2016.

Ponte, J. et al. (2016): UDIAF. Servizos comunitarios de Atención Temperá. Ferrol, Galicia: Asociación Galega de Atención Temperá (en línea). <https://www.researchgate. net/publication/3045268Io_UNIDADES_ DE_DESENVOLVEMENTO_INFANTIL_E_ APOIO_FAMILIAR_SERVIZOS_ COMUNITARIOS_DE_ATENCION_ TEMPERA>, acceso I de septiembre de 2016.

Ponte, J. (2015). "Las dificultades de clasificación diagnóstica en atención temprana. La experiencia de dos unidades en Galicia”. Revista Española de Discapacidad, 3 (I): I23I39.

Ponte, J et al. (2004): Guía de Estándares de Calidad en Atención Temprana. Madrid: Imserso.

Red de Consultoría Social (2010): Guía para la gestión con indicadores en acción social. Madrid: Ministerio de Sanidad, Politica Social e Igualdad (en línea). <http://www. indicadoressociales.org/Archivos/Documentos/ Secciones/74_es-ES_Gu $\% \mathrm{C}_{3} \% \mathrm{ADa} \% 20$ documento \% 2ocompleto.pdf>, acceso I de septiembre de 2016 .
Romero, R. (20I I): Evaluación de la calidad percibida en los centros de atención infantil temprana de la provincia de Málaga (tesis doctoral). Universidad de Málaga (en línea). $<$ https://dialnet.unirioja.es/servlet/tesis?codigo $=2420 \mathrm{I}$ \&orden $=329895$ \&info=link $>$, acceso I de septiembre de 2016 .

Rubert, M. A (20I0): "La calidad de los centros y la evaluación”, en Cristobal, C. et al. (coords.): La atención temprana. Un compromiso con la infancia y sus familias. Barcelona: Editorial UOC.

Soriano V. y Kyriazopoulou, M. (2010): Early Childhood Intervention: Progress and Developments. Bruselas: European Agency for Development and Education in Special Needs Education.

Soriano, V. (2005): Atención Temprana en Europa. Aspectos clave y recomendaciones. Bruselas: European Agency for Development and Education in Special Needs Education.

VV.AA. (I999): "Evaluation of Child Disability Services". Child Care, Health and Development, 25 (2): 8I-I73 (en línea). $<$ http://onlinelibrary.wiley.com/doi/Io.I I I I/ cch.I999.25.issue-2/issuetoc.>, acceso Io de mayo de 2016 .

Xunta de Galicia. Decreto I83/2013, de 5 de diciembre, por el que se crea la Red Gallega de Atención Temprana, Diario Oficial de Galicia, 23 de diciembre de 20I3, núm. 244, pp. 49324-49344.

Xunta de Galicia (2015): Protocolo conjunto de Coordinación, Derivación e Intervención en Atención Temprana (en línea). <http:// benestar.xunta.es/export/sites/default/Benestar/ Biblioteca/Documentos/Contidos_Estandar/ Protocolo_Atencion_tempera_GAL.pdf>, acceso I de septiembre de 20 I 6 . 